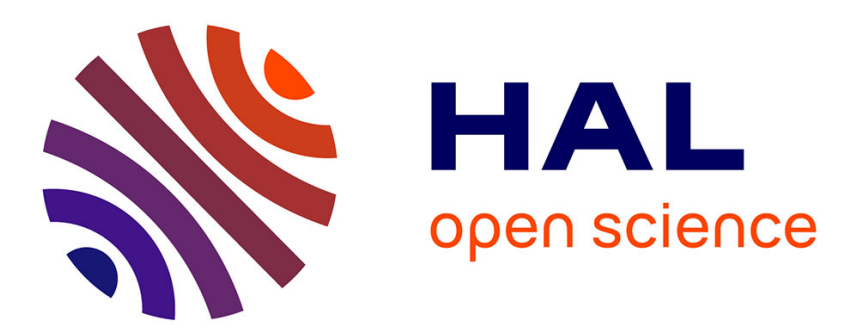

\title{
Visible and near infrared optical properties of small particle random composites : carbon black in $\mathrm{KBr}$
}

\author{
D. Ordiera, F. Carmona
}

\section{To cite this version:}

D. Ordiera, F. Carmona. Visible and near infrared optical properties of small particle random composites: carbon black in KBr. Journal de Physique, 1983, 44 (6), pp.683-689. 10.1051/jphys:01983004406068300 . jpa-00209647

\section{HAL Id: jpa-00209647 https://hal.science/jpa-00209647}

Submitted on 1 Jan 1983

HAL is a multi-disciplinary open access archive for the deposit and dissemination of scientific research documents, whether they are published or not. The documents may come from teaching and research institutions in France or abroad, or from public or private research centers.
L'archive ouverte pluridisciplinaire HAL, est destinée au dépôt et à la diffusion de documents scientifiques de niveau recherche, publiés ou non, émanant des établissements d'enseignement et de recherche français ou étrangers, des laboratoires publics ou privés. 
Classification

Physics Abstracts

78.20D

\title{
Visible and near infrared optical properties of small particle random composites : carbon black in $\mathrm{KBr}$
}

\author{
D. Ordiera and F. Carmona \\ Centre de Recherches Paul Pascal, Domaine Universitaire, 33405 Talence, France
}

(Reçu le 6 octobre 1982, accepté le 21 février 1983)

\begin{abstract}
Résumé. - Nous présentons des résultats de mesures spectroscopiques entre 0,3 et $1,8 \mu$ sur des composites noir de carbone dans une matrice de $\mathrm{KBr}$. Nous montrons que les théories du milieu effectif rendent correctement compte de nos résultats aux faibles concentrations en noir de carbone. En revanche, aux concentrations supérieures à quelques pourcents, ces théories ne peuvent interpréter les deux faits expérimentaux essentiels déduits de nos résultats : existence à toute longueur d'onde d'un maximum prononcé de la courbe de réflectivité en fonction de la concentration $\phi$ au voisinage de $\phi=0,50$; réflectivité indépendante de la longueur d'onde, c'est-à-dire des propriétés optiques du noir de carbone pur, jusqu'à $\phi=0,40$. La possibilité d'une interprétation de ces résultats par la théorie de la percolation est discutée.
\end{abstract}

\begin{abstract}
Spectroscopic data obtained between 0.3 and $1.8 \mu$ on composites constituted by carbon black particles in a $\mathrm{KBr}$ matrix are reported as functions of wavelength and carbon volume concentration $\phi$. We show that effective medium theories account correctly for the experimental results at very low concentrations. At concentrations higher than a few percent, these theories fail to interpret the two striking experimental facts deduced from the results : the existence, at eyery wavelength, of a maximum in the concentration dependence of the reflectivity and the fact that the reflectivity does not depend on wavelength, that is on carbon reflectivity, up to $\phi=0.40$. These facts are tentatively attributed to percolative effects.
\end{abstract}

\section{Introduction.}

The propagation of an electromagnetic wave in an inhomogeneous medium or in a medium containing particles is a problem more than one hundred years old [1-3]. The general solution, for light scattering by an isolated particle has long been established : it is known as Mie's theory [1, 2]. However, this problem is still up to date on both theoretical and practical grounds. On the one hand, so far, no good theory exists which takes correctly into account the interactions between particles when their concentration in the medium is high. On the other hand, it has been experimentally evidenced that this class of materials with metallic particles exhibits spectral selectivity of the kind required in solar energy conversion $[4,5]$.

When the particles are much smaller than the wavelength, two effective medium theories have been proposed : the so-called effective medium theory of Bruggeman [6] (EMT) which is self-consistent and the non self-consistent theory of Maxwell Garnett (MG) [7]. The calculations are performed in the quasi static limit (the instantaneous fields inside the particle are constant), the dielectric permittivities involved in the formulae being in general complex numbers. These approaches considering an effective dielectric permittivity for the composite are meaningful when the material actually behaves like a homogeneous body (in particular, the overall light scattering is negligible [8]). For very low particle concentrations, both theories give identical results, often in agreement with experiments. When concentrations are higher than a few percent, they generally give different results, often in disagreement with experiments.

Numerous corrections have been proposed to take into account effects not included in the original theories : magnetic dipole effect [9], non homogeneous particles (for example oxide layers [10]), particle size distribution [10], non spherical particles [11, 12], existence of agglomerates $[11,13], \ldots$ These corrections lead to introduce parameters in the calculations, the actual value of which is unknown, so that they are adjusted by fitting the experimental data. Some agree- 
ment is then found by using either of the theories and from numerous works published in this field it is clear that the two theories in fact describe materials with different structures $[14,15]$ : the MG theory seems to apply better to particle composites whereas the EMT describes solids in which the constituents have to be considered on the same grounds.

A completely different approach describes the propagation of the wave through the heterogeneous medium by means of perturbative methods [16] or by considering multiple scattering [17]. To the best of our knowledge, these theories have not yet been applied to experimental data.

Neither of these theories however, despite a few phenomenological [12] or theoretical [18] attempts, really takes into account the microstructure of the media. It is indeed known that, when the medium is truly random, the particles are allowed to form clusters the mean size of which increases with concentration, an infinite cluster existing above a critical concentration. This clustering is of crucial importance for the electrical properties of metal-insulator composites for which the percolation theory, which precisely describes the connectivity of the particles, gives, with some definite restrictions, a good interpretation [19]. One has then to ask the question : what is, if any, the influence of clustering on the optical properties ? Such percolative effect could eventually exist since it has been theoretically predicted $[20,21]$ and experimentally confirmed [22] that the dielectric constant of conductor-insulator composites exhibits anomalous behaviours that are not deduced from effective medium theories.

In order to investigate experimentally the eventual links between percolation and the optical properties, model composites with precisely known composition, structure and intrinsic properties of the pure constituents are needed. Good candidates are pelletized particles in a $\mathrm{KCl}$ matrix composites as has been recently shown $[23,24]$.

We will report in this article spectroscopic data obtained in the visible and near infrared ranges on similar composites : carbon black particles in a $\mathrm{KBr}$ matrix. The structure and properties of carbon blacks are well-known [25]. They are often spherical particles' with reasonably uniform diameters in a given black that can be varied over a wide range (10 to $500 \mathrm{~nm}$ ) depending on preparation conditions. Their optical constants vary slowly in the wavelength range we are considering; they can be modified by means of thermal treatment at temperatures higher than preparation temperature. Also, $\mathrm{KBr}$ is a dielectric without losses and its optical index remains fairly constant in the same wavelength range.

In the first series of experiments that we report here we have chosen a black with particles much smaller than the wavelength. The varying parameter is then the volume concentration of carbon particles.

This article is organized as follows : we report in section 2 the preparation and characterization of the samples and the spectroscopic methods; the experimental results are given in section 3 and discussed in section 4, and we will conclude in section 5 .

\section{Experimental methods.}

The carbon black used in this study was obtained from the Cabbot Co. It is known under the name Monarch 1100 , the mean particle diameter is $16 \mathrm{~nm}$ and the electrical conductivity measured on compressed powder is $10 \Omega^{-1} \mathrm{~cm}^{-1}$. The $\mathrm{KBr}$ used is of spectroscopic quality. The samples are made by means of the classical method used in infrared spectroscopy. The two constituents are carefully mixed in a mortar; the resulting powder is ground in a mechanical grinder, evacuated at a pressure lower than $10^{-3}$ torr and pelletized with a pressure of 8 tons $/ \mathrm{cm}^{2}$. The pistons used for pelletizing are made of tungsten carbide and were frequently mechanically polished. Typical pellets have $12 \mathrm{~mm}$ diameter and $0.2 \mathrm{~mm}$ thickness.

The first problem to be solved in the preparation is a uniform distribution of particles. It is well-known [25] that carbon black particles can form agglomerates with variable sizes. The pellets were thus repeatedly broken and re-pelletized several times until perfect reproducibility of the optical spectrum was obtained. The mean number of cycles was found to be 10 to 12 . We verified, by electronic microscopy on very lightly concentrated samples $(\phi<0.1 \%)$, that the spatial distribution of particles is fairly uniform and that agglomerates are almost totally absent.

The volume concentration in particles was determined by weighing the constituents. After the successive preparation cycles, we verified the final concentration by density measurements : it always agreed with initial concentration within $1 \%$. The smallest concentrations were obtained by diluting more concentrated samples $(5 \%)$.

It was also required that the composites be random. As a characterization of this series of samples, we performed electrical resistance measurements. As expected, a conductivity threshold was evidenced, the critical concentration being $\phi^{*}=0.16$. This figure is in good agreement with previously reported conductivity threshold for random 3D metal-insulator composites [26].

Great care was taken while handling the samples due to possible pollution by water vapour which gives rise to strong light scattering.

Reflection and, whenever possible, transmission spectra were recorded between 300 and $1800 \mathrm{~nm}$ with a PERKIN ELMER 330 spectrophotometer with an integrating sphere attachment. For every sample the hemispherical and quasi normal (incident angle $11^{\circ}$ ) reflectivities and, depending on samples, the total and diffuse transmission were measured. We verified that the diffuse component represented not more than $5 \%$ of the total reflection and transmission. 
In a few case, by varying the thickness of pellets of a given carbon concentration we verified that the Beer-Lambert law for light absorption was obeyed. This fact together with the weak diffusion enables us to state that, at least at very low concentrations, the composites can be described by effective optical constants $n$ and $k$ as if they were homogeneous bodies.

\section{Experimental results.}

3.1 Optical CONSTANTS OF THE CONSTITUENTS. The optical constants of pure $\mathrm{KBr}$ have been determined by means of measurements of the reflection and the transmission factors $R$ and $T$ of a pellet and use of the results of classical optics as recalled in the appendix. It has been verified that the extinction coefficient $k$ is zero to within experimental uncertainty. The values of the refractive index $n_{0}$ for various wavelengths are given in table $I$ : they are in agreement with values of the literature [27].

Table I. - Experimental optical constants at four wavelengths of $\mathrm{KBr}$ and carbon black.

$\begin{array}{lllll}\lambda & 400 & 600 & 1000 & 1600 \\ \overline{n_{0}} & \overline{-} & -\overline{6} & - & - \\ n_{\mathrm{c}} & 1.23 & 1.581 & 1.568 & 1.559 \\ k_{\mathrm{c}} & 0.454 & 0.455 & 1.312 & 1.553 \\ & & & 0.559 & 0.649\end{array}$

As carbon is strongly absorbing in the visible and infrared, we deduced its optical constants $n_{\mathrm{c}}$ and $k_{\mathrm{c}}$ from the reflectivity measurements on a pellet of pure $\mathrm{KBr}$, one of its faces being coated under pressure with a continuous layer of carbon black.

As recalled in the appendix, the optical constants of the carbon coating can be calculated from the measurements of the reflection factors of the $\mathrm{KBr}$ plate, the reflection coefficient of the coating in air and the knowledge of the refractive index of $\mathrm{KBr}$.

In figure 1, we have reported the reflection spectrum between 300 and $1800 \mathrm{~nm}$ of carbon in air (dashed line). As expected $\rho$ is. low and increases strongly with $\lambda$. In table I, we have given the experimental values of $n_{\mathrm{c}}$ and $k_{\mathrm{c}}$ for various wavelengths. These are average values of measurements on several pellets. The experimental uncertainty is about $2 \%$ on $n$, and about $4 \%$ on $k$. We notice that $n_{\mathrm{c}}$ varies slowly whereas $k_{\mathrm{c}}$ increases strongly with $\lambda$. These values are in agreement with previously reported carbon black optical constants [28].

3.2 WeAK CONCENTRATIONS. - For carbon concentrations lower than $0.3 \%$ the pellets transmit light so that the optical constants $n$ and $k$ of the composite can be calculated. As an example, the experimental reflection and transmission spectra of a sample containing $0.125 \%$ of carbon are given in figure 2 . The transmission spectrum is strongly wavelength dependent. In this concentration range $n$ is found,

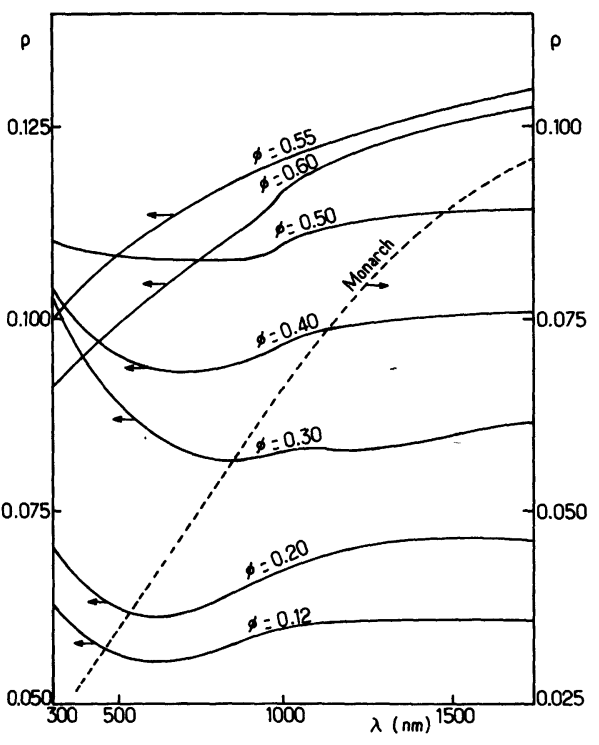

Fig. 1. - Hemispherical reflectivities of carbon black/KBr composites at various carbon concentrations between 0.3 and $1.8 \mu$. Dashed line : hemispherical reflectivity of pure carbon.

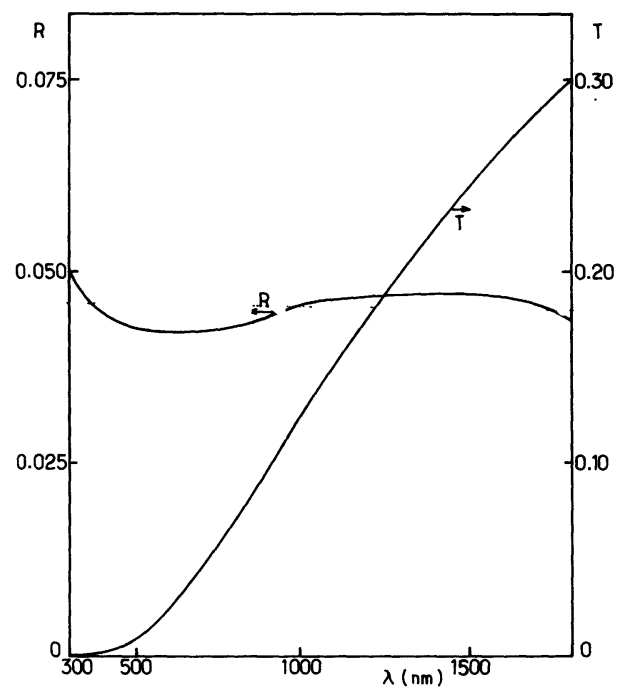

Fig. 2. - Hemispherical reflectivity and transmission factors of a slob of composite with carbon concentration $\phi=0.00125$ between 0.3 and $1.8 \mu$.

within experimental uncertainty, to be identical to $n_{0}$. In figure 3 we have plotted the concentration dependence of the absorption coefficient $\alpha$ at three wavelengths : a linear relationship between $\alpha$ and $\phi$ is evidenced. In figure 4 we have plotted the deduced concentration dependence of the extinction coefficient $k$ : within experimental errors, $k$ seems to be wavelength independent.

3. 3 High CONCENTRATIONS. - Due to their thickness, the pellets do not transmit light when the concentration is higher than $0.5 \%$. In figure 1 , we have reported the hemispherical reflectivity of various samples 
with concentrations increasing from 12 to $60 \%$. Up to $\phi=0.50$ the reflectivity is essentially $\lambda$ independent as is that of $\mathrm{KBr}$. But above $50 \%$, a strong $\lambda$ dependence of the reflectivity appears suddenly and the spectra resemble that of pure carbon. We also notice that the composite reflectivity is higher than the reflectivities of either constituent over a wide range of values of $\phi$.

Figures 5, 6, 7 and 8 are plots of the concentration dependence of the composite reflectivity at four wavelengths. The values at $\phi=1$ correspond to measured reflectivities on pure carbon. The existence of a maximum at each wavelength is evidenced and we notice that the height and position of this maximum depends on $\lambda$.

\section{Discussion.}

As we recalled in the introduction, effective medium theories are widely used for interpreting the optical properties of heterogeneous solids. The effective permittivity of the composite is given by the relations M.G. theory

$$
\varepsilon=\varepsilon_{\mathrm{m}} \frac{\varepsilon_{\mathrm{n}}(1+2 \phi)+2 \varepsilon_{\mathrm{m}}(1-\phi)}{\varepsilon_{\mathrm{n}}(1-\phi)+\varepsilon_{\mathrm{m}}(2+\phi)}
$$

EMT

$$
\begin{gathered}
\varepsilon=f \varepsilon_{\mathrm{n}} \\
\text { with }\left\{\begin{aligned}
f & =a+\sqrt{a^{2}+x / 2} \\
a & =\frac{1}{4}|(3 \phi-1)(1-x)+x| \\
x & =\frac{\varepsilon_{\mathrm{m}}}{\varepsilon_{\mathrm{n}}}
\end{aligned}\right.
\end{gathered}
$$

$\varepsilon_{\mathrm{m}}$ being the permittivity of the matrix and $\varepsilon_{\mathrm{n}}$ that of the particles, the concentration of which is $\phi$. Both $\varepsilon_{\mathrm{n}}$ and $\varepsilon_{\mathrm{m}}$ are allowed to be complex and are related to $n$ and $k$ by the classical relations

$$
\left\{\begin{aligned}
\varepsilon & =\varepsilon^{\prime}-j \varepsilon^{\prime \prime} \\
\varepsilon^{\prime} & =n^{2}-k^{2} . \\
\varepsilon^{\prime \prime} & =2 n k
\end{aligned}\right.
$$

Notice that there is mathematically a problem in the square root determination in (2); in fact only one solution is physically meaningful.

We have compared the predictions of both theories with our experimental results. .We want to emphasize the fact that all figures involved in the theoretical relations are directly deduced from the experiments. For weakly concentrated samples direct comparison is allowed on $n$ and $k$, whereas for the more concentrated samples we compare the calculated and the experimental reflectivities.

4.1 WEAKLY CONCENTRATED SAMPLES. - In figure 3 the calculated values of $\alpha$ at the three wavelengths used in the experiments. At these very low concentrations both theories give the same linear concentration

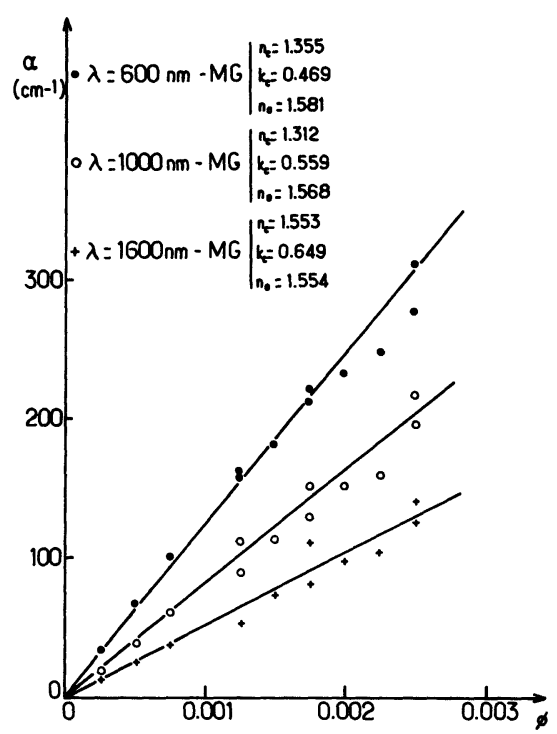

Fig. 3. - Concentration dependence of the absorption coefficient $\alpha$ at three wavelengths : 600,1 000 and $1600 \mathrm{~nm}$. Straight lines : theoretical values for $\alpha$ as given by the effective medium theories using the as reported optical constants of the constituents.

dependence. There is good agreement between the experimental and calculated values of $\alpha$. As a consequence, we notice that both theories account for the wavelength independence of $k$ as shown in figure 4 . On the other hand, in this concentration range both theories predict a slight difference between $n$ and $n_{0}$, that is less than the experimental uncertainty.

The good agreement found between these theories and experiments is satisfactory since no adjustable parameters have been used. It also confirms that effective medium theories are better the lower the concentration in one constituent.

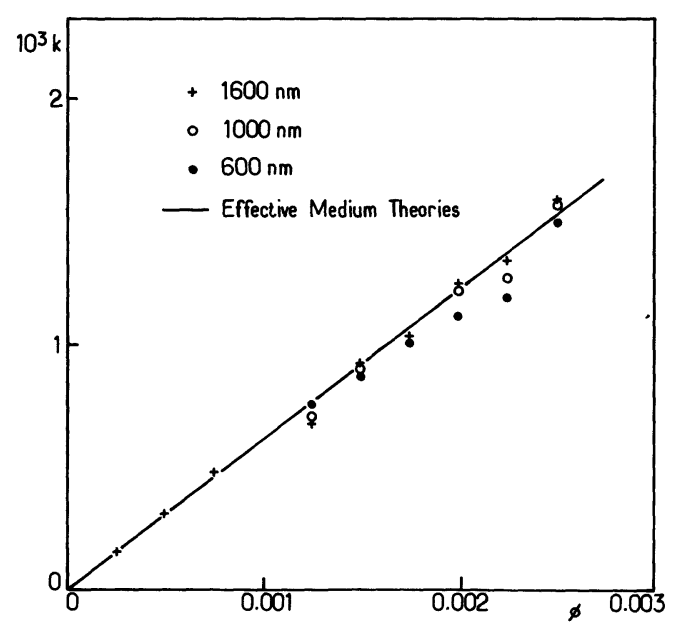

Fig. 4. - Concentration dependence of the extinction coefficient $k$ at the same three wavelengths as in figure 3. Straight line is given by effective medium theories. 
4. 2 Highly CONCENTRATED SAMPLES. - The concentration dependences of the reflectivity at the four experimental wavelengths as calculated by the effective medium theories are plotted in figures 5 to 8 . Both theories give nearly the same results. Clearly neither theory accounts for the experimental results. In particular, they do not predict any maximum. On the contrary, they both predict a minimum for the lowest wavelengths. We also calculated in a few cases the bounds proposed independently by Bergman [29] and Milton [30] for $\varepsilon$. In all cases the experimental values fall well outside these bounds.

We have noticed the great similarities between the reflectivity versus concentration curves at the various wavelengths. On the other hand, we also noticed the (small) variation of the refractive index $n_{0}$ of pure $\mathrm{KBr}$ in the spectral range. It would be extremely useful to correct our data for these $n_{0}$ variations. Such a correction should be made directly on the composites $n$ and $k$ values which are not presently known. As a rough correction, we have subtracted, from the measured reflectivities $\rho$, the pure $\mathrm{KBr}$ reflectivity $\rho_{0}$ at the corresponding wavelength. The values of $\rho-\rho_{0}$ for all four wavelengths versus the

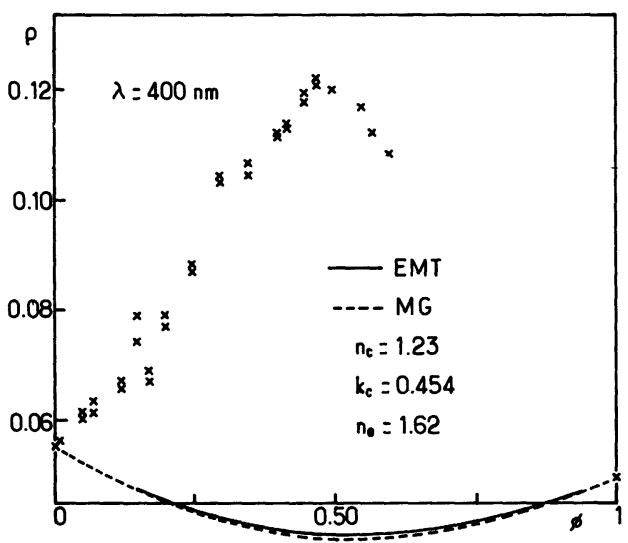

Fig. 5. - Concentration dependence of the hemispherical reflection coefficient at $\lambda=400 \mathrm{~nm}$. Full line and dashed line are given by EMT and MG theories respectively.

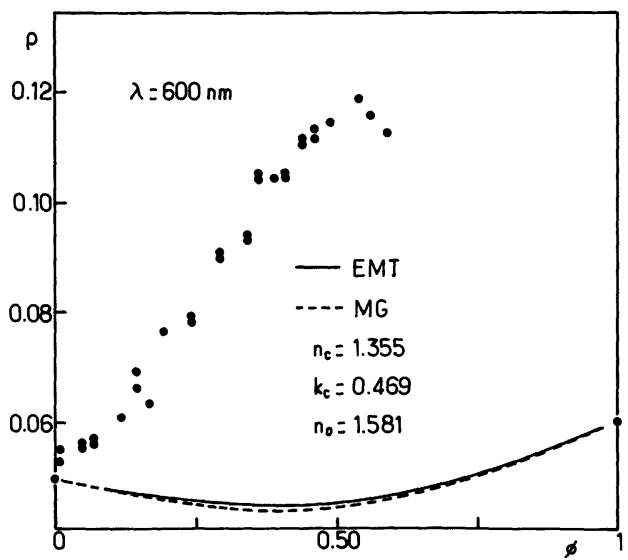

Fig. 6. - Same as figure 5 at $\lambda=600 \mathrm{~nm}$.

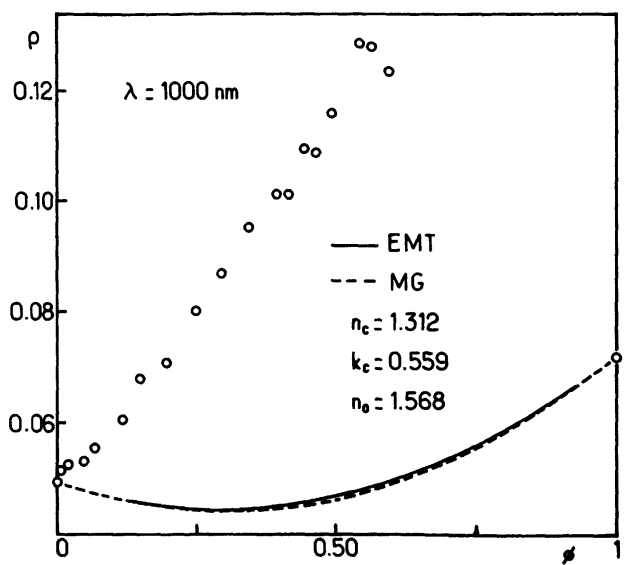

Fig. 7. - Same as figure 6 at $\lambda=1000 \mathrm{~nm}$.

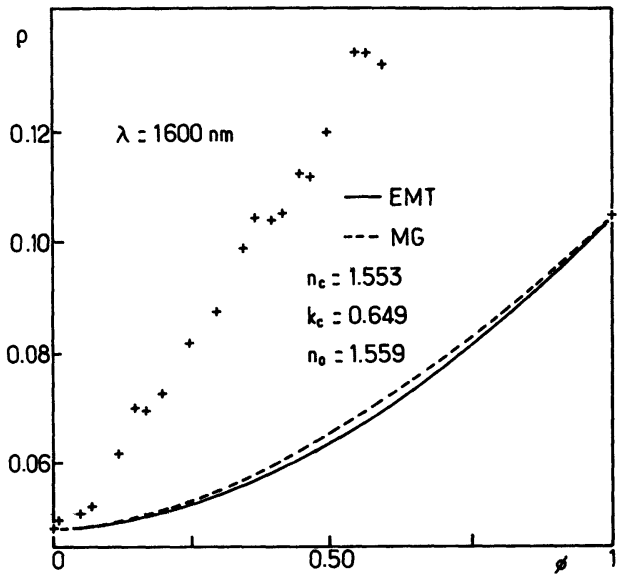

Fig. 8. - Same as figure 7 at $\lambda=1600 \mathrm{~nm}$.

concentration are plotted in figure 9. Within experimental errors, the points fall on a single curve up to $\phi=0.40$. Beyond this value the curves tend to separate, extrapolating to the pure carbon " corrected " reflectivity which is strongly wavelength dependent.

Although such representation of our data has no real physical meaning, it qualitatively reproduces the observed variations of the reflection spectra with concentration : "pure $\mathrm{KBr}$-like» spectra at low concentrations, "pure carbon-like" spectra at high concentrations. The existence of a wavelength independent behaviour of $\rho-\rho_{0}$ over a large concentration range is not accounted for by EMT and MG theories.

In insulator-conductor mixtures, percolation theory predicts $[20,21]$, in the static limit, a divergence of the real part of the dielectric permittivity at percolation threshold. According to Efros and Shklovskii [20] below the critical concentration the permittivity of the composite depends only on the concentration and the permittivity of the dielectric matrix. Such a fact could account for a wavelength independent reflectivity that we observed. However, little is known about the critical behaviour of the electrical permittivity at non zero frequencies. According to Efros and Shklovskii [20], one should observe a maximum 


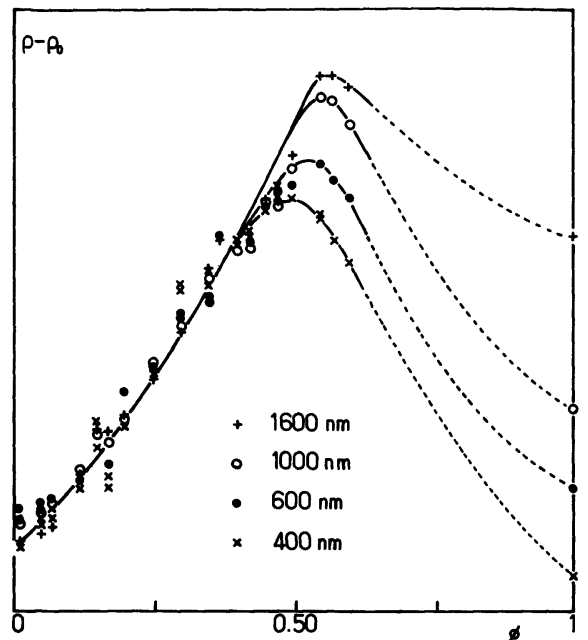

Fig. 9. - Concentration dependence of the hemispherical reflection coefficient of the composites corrected for the reflectivity of pure $\mathrm{KBr}, \rho-\rho_{0}$, at the four wavelengths. Full and dashed lines are guides for the eye.

instead of a divergence at $p_{\mathrm{c}}$. Microwave measurements [22] showed that the anomaly in the dielectric constant is moved towards concentrations higher than d.c. conductivity threshold, presumably due to dimensionality effects (small penetration of the microwave field). At optical frequencies, we could expect the dielectric anomaly to subsist, the concentration at which it should occur being still unknown. We can notice here that in the actual experiments the penetration depth of optical radiations is about 4 to 12 times the particle diameter, depending on wavelength, over the experimental range. Then dimensionality effects must not be excluded.

However, the observed discrepancies between our results and the predictions of the effective medium theories are puzzling since it is largely admitted that, despite the approximations involved in the effective medium theory, it should reproduce percolative effects if they do exist in our materials. As no adjustable parameters are used in the calculations, one might question the values of the optical constants of carbon. As there is good agreement between experimental and calculated values at low. concentrations, this would mean that, for some unknown reason, these constants in the composite change with concentration. Considering the preparation conditions of the samples, we believe this does not occur. However, one may question the actual structure of the composites at large concentrations especially at and near the surface. The possibility of an ordered arrangement of the particles leading to diffraction effects might have to be considered.

\section{Conclusion.}

We have shown that the usually used EMT and MG theories both account for the optical properties of weakly concentrated carbon black in $\mathrm{KBr}$ composites. They both fail to account for the experimental reflectivity at higher concentrations. The most striking fact that emerges from this study is that the reflectivity of the composite is, in a given concentration range, much higher than both reflectivities observed on the pure constituents. Neither the effective medium theories nor the bounds given for the permittivity of heterogeneous materials predict this possibility. On the other hand, we recalled that percolation theory predicts a dielectric constant anomaly which could lead to this peculiar reflectivity behaviour. However, before deciding, more has to be known on theoretical grounds on this anomaly at high frequencies (microwave and optical) and on experimental grounds on the actual behaviour of the optical constants of the composites and on their actual structure at large concentrations. Work is in progress to determine $n$ and $k$ independently and will be published elsewhere.

\section{Appendix.}

Reflection and transmission factors $R$ and $T$ of a plane parallel plate of thickness $e$ under normal incidence and their inversion for calculating the optical constants of materials.

1. Absorbing material in air. - With $n-j k$ being the complex refractive index of the material, the reflection coefficient on one face of the plate is :

$$
\rho=\frac{(n-1)^{2}+k^{2}}{(n+1)^{2}+k^{2}} .
$$

The reflection and transmission factors $R$ and $T$ of the plate are :

$$
\begin{aligned}
& R=\rho\left|1+\frac{(1-\rho)^{2} \tau^{2}}{1-\rho^{2} \tau^{2}}\right| \\
& T=\frac{(1-\rho)^{2} \tau}{1-\rho^{2} \tau^{2}}
\end{aligned}
$$

where $\tau=\exp (-\alpha e)($ A.4) as given by Beer-Lambert's Law, $\alpha$ being the absorption coefficient

$$
\alpha=\frac{4 \pi k}{\lambda}
$$

$\rho$ and $\tau$ are obtained from $R$ and $T$ by inverting (A.2) and (A.3)

$$
\begin{gathered}
\rho=\frac{-\left(R^{2}-2 R-1-T^{2}\right)-\left|\left(R^{2}-2 R-1-T\right)^{2}-4 R(2-R)\right|^{1 / 2}}{2(2-R)} \\
\tau=\frac{R-\rho}{\rho T} .
\end{gathered}
$$


$k$ is deduced directly from (A.4) and (A.5) and $n$ is being in air, is

obtained from (A.6) and (A.1).

2. - Non absorbing plate $\left(n_{0}\right)$ coated on one side with a totally absorbing layer $\left(n_{0}-j k_{0}\right)$ in air. Let $\rho_{0}$ be the reflection coefficient of the uncoated face of the plate, and $\rho_{1}$ the reflection coefficient of the coated face, the incident beam being in the medium $n_{0}$

$$
\rho_{1}=\frac{\left(n_{\mathrm{c}}-n_{0}\right)^{2}+k_{\mathrm{c}}^{2}}{\left(n+n_{0}\right)^{2}+k_{\mathrm{c}}^{2}} .
$$

The reflection factor of the plate, the incident beam

$$
R=\rho_{0}+\frac{\rho_{1}\left(1-\rho_{0}\right)^{2}}{1-\rho_{1} \rho_{0}} .
$$

Knowing $R$ and $\rho_{0}, \rho_{1}$ is deduced from (A.9). If the reflection coefficient $\rho_{2}$ of the coating in air

$$
\rho_{2}=\frac{\left(n_{\mathrm{c}}-1\right)^{2}+k_{\mathrm{c}}^{2}}{\left(n_{\mathrm{c}}+1\right)^{2}+k_{\mathrm{c}}^{2}}
$$

is measured, $n_{\mathrm{c}}$ and $k_{\mathrm{c}}$ are deduced from both (A.8) and (A.10), $n_{0}$ being known.

\section{References}

[1] VAN DE HULST, H. C., Light scattering by small particles (Wiley, New York) 1957.

[2] KERKER, M., The scattering of light (Academic Press, New York) 1969.

[3] See for example, Electrical transport and optical properties of inhomogeneous media, J. C. Garland and D. B. Tanner ed. (AIP, New York) 1978.

[4] Craighead, H. G., Bartynski, R., Buhrman, R. A., WoJCIK, L. and Sievers, A. J., Sol. En. Mat. 1 (1979) 105.

[5] Spitz, J., Aubert, A., Behagel, J. R., Berthier, S., LAfait, J. and J. Rivory, Revue Phys. Appl. 14 (1979) 67.

[6] Bruggeman, D. A. G., Ann. Phys. (Leipzig) 24 (1935) 636.

[7] Maxwell Garnett, J. C., Philos. Trans. R. Soc. London 203 (1904) 385; 205 (1906) 237.

[8] Berthier, S., Thèse de 3e cycle, Paris VI, 1979.

[9] Stroud, D. and Pan, F., Phys. Rev. B 17 (1978) 1602.

[10] Woop, D. M. and Ashcroft, N. N., Philos. Mag. 35 (1977) 269.

[11] Granqvist, C. G. and Hunderi, O., Phys. Rev. B 16 (1977) 3513.

[12] Cohen, R. W., Cody, C. D., Coutts, M. D. and Abeles, B., Phys. Rev. B 8 (1973) 3689.

[13] Clippe, P., Evrard, R. and Lucas, A. A., Phys. Rev. $B 14$ (1976) 1715.

[14] Gittleman, J. I. and Abeles, B., Phys. Rev. B 15 (1977) 3273.

[15] Gibson, U. J., Craighead, H. G. and Buhrman, R. A., Phys. Rev. B 25 (1982) 1449.
[16] Briggs, J. and Schwartz, L., Phys. Rev. A 16 (1977) 1199 ;

Briggs, J., Phys. Rev. A 18 (1978) 1577.

[17] Lamb, W., Wood, D. M. and AshCroft, N. N., Phys. Rev. B 21 (1980) 2248.

[18] Ping Sheng, Phys. Rev. Lett. 45 (1980) 60.

[19] Kirkpatrick, S., Rev. Mod. Phys. 45 (1973) 574.

[20] Efros, A. L. and ShklovskiI, B. I., Phys. Status Solidi $B 76$ (1976) 475.

[21] Bergmann, D. J. and Imry, Y., Phys. Rev. Lett. 39 (1977) 1222.

[22] Clerc, J. P., Thèse d'Etat, Marseille, 1980.

[23] Grannan, D. M., Garland, J. C. and Tanner, D. B., Phys. Rev. Lett. 46 (1981) 375.

[24] Russel, N. E., Garland, J. C. and Tanner, D. B., Phys. Rev. B 23 (1981) 632;

Carr, G. L., Henry, R. L., Russell, N. E., Garland, J. C. and TANner, D. B., Phys. Rev. B 24 (1981) 777.

[25] Donnet, J. B. and Voet, A., Carbon Black (Dekker, New York) 1976.

[26] Webman, I., Jortner, J. and Cohen, M. H., Phys. Rev. B 11 (1975) 2885;

Fug, G., Canet, R. and Delhaes, P., C.R. Hebd. Séan. Acad. Sci. Paris B 287 (1978) 5.

[27] Handbook of Chemistry and Physics, The Chemical Rubber Co. Ed. (Cleveland) 1966.

[28] Ravey, J. C., Thèse d'Etat, Université de Nancy, 1973.

[29] Bergman, D. J., Phys. Rev. Lett. 44 (19), (1980) 1285.

[30] Milton, G. W., Appl. Phys. Lett. 37(3) (1980) 300. 